\title{
Exact and asymptotic scaling solutions for fragmentation with mass loss
}

\author{
Mao Cai, Boyd F. Edwards, and Hongtao Han \\ Department of Physics, West Virginia University, Morgantown, West Virginia 26506
}

(Received 12 December 1988; revised manuscript received 16 July 1990)

\begin{abstract}
Exact and asymptotic solutions to a linear rate equation for fragmentation with mass loss are presented. Solutions for spatially discrete random bond annihilation illustrate the mutual exclusiveness of the fragmentation and recession terms in the rate equation. Exact solutions for deterministic equal fragment recession show that continuous mass loss between fragmentation events can be approximated by discrete mass loss during fragmentation events when this mass loss is small. Evidence that continuous and discrete mass loss preclude shattering mass loss, the loss of mass to zeromass particles due to runaway fragmentation, is presented. General asymptotic scaling forms, general solutions reflecting arbitrary initial conditions, and explicit scaling solutions are derived.
\end{abstract}

\section{INTRODUCTION}

The physics of fragmentation for mass-conserving systems has attracted recent attention. Dynamic scaling relations have been derived explicitly for random scission of one-dimensional chains ${ }^{1}$ and have been shown to exist for general scission processes. ${ }^{2}$ Scission rates which diverge in the limit of small particles yield "shattering," runaway fragmentation producing unexpected loss of mass to zero-mass particles. ${ }^{3}$ Random scission concepts grew out of polymer breakup applications and agree with experimental data on comminution (grinding). ${ }^{4}$

Physical systems which do not conserve solid mass during fragmentation abound. Oxidation, melting, sublimation, and dissolution cause the exposed surface of a porous solid particle to recede continuously, eventually leading to total loss of the solid mass of the particle. This surface recession can widen the pores of a solid, causing loss of connectivity and fragmentation as the pores fuse [Fig. 1(a) $-1(\mathrm{c})$ ]. Thus, instead of requiring a mechanism for discrete cutting events as for random scission, this fragmentation arises from the continuous process of surface recession; when surface recession removes the last link between two or more pieces, a mass-conserving fragmentation event occurs. Experiments indicate that hundreds of such fragmentation events can occur during the oxidation of a single coal-char particle. ${ }^{5}$

Systems also exist for which mass loss is discrete. For a reactive solid containing isolated explosive regions, non-mass-conserving explosive fragmentation events punctuate the continuous surface recession process [Fig. 1(d) $-1(f)]$. These fragmentation events arise from the expansion of the gases created from the (effectively instantaneous) reaction of the solid mass of explosive regions, rather than the loss of connectivity due to continuous surface recession. A heterogeneous reactive solid with two nonexplosive phases, one of which reacts much faster than the other, also yields effectively discrete mass loss when the fast phase reacts and causes loss of connectivity of the solid.

This paper extends the theory of linear fragmentation with mass $\operatorname{loss}^{6}$ by deriving general asymptotic scaling forms and exact solutions. Solutions for random, deterministic, and spatially discrete systems illustrate a spectrum of applications of the theory, and illuminate the nature of dynamic scaling. Conditions for "shattering" mass loss to zero-mass particles due to runaway fragmentation are discussed. Although applications to surface recession processes are most apparent to us, the theory nevertheless applies unchanged to general phenomena in which the removal of solid mass leads to fragmentation, whether the mass is removed from the surface or from the interior of the object. Simulations for surface mass removal have been performed by Sahimi and Tsotsis. ${ }^{7}$

In Sec. II, a particle current relevant to the linear rate equation for fragmentation with mass loss is introduced, and a correspondence with the equations for (massconserving) polymer breakup is made. One-dimensional discrete-space problems including random scission and random bond annihilation are studied in Sec. III. A deterministic equal-fragment recession problem is defined in Sec. IV and solved exactly for both continuous and discrete mass loss. Asymptotic dynamic scaling solutions, explicit solutions, and shattering are discussed in Sec. V. Finally, conclusions and future directions are outlined in Sec. VI.

\section{RATE EQUATION} loss, ${ }^{6}$

$$
\begin{aligned}
\frac{\partial n(x, t)}{\partial t}= & -a(x) n(x, t)+\int_{x}^{\infty} a(y) \bar{b}(x \mid y) n(y, t) d y \\
& +\frac{\partial}{\partial x}[c(x) n(x, t)],
\end{aligned}
$$

involves a particle mass distribution $n(x, t)$, a fragmentation rate $a(x)$, a distribution $\bar{b}(x \mid y)$ of particle masses $x$ spawned by the fragmentation of a particle of mass $y$, and a continuous mass loss rate $c(x)$, defined so that $c(m(t))=-d m / d t$ for a particle of time-dependent mass $m(t)$. The terms on the right side of Eq. (1) describe, from left to right, the reduction in the number of particles in the mass range $[x: x+d x]$ due to the fragmenta- 
(a)

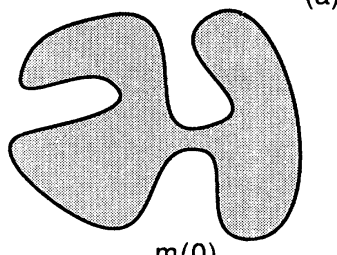

(b)
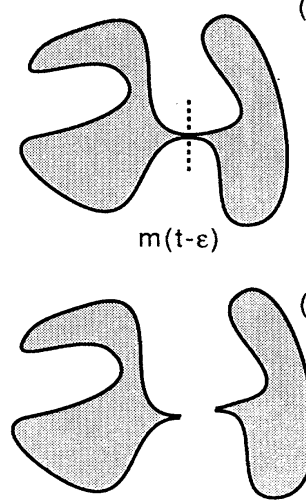

$m_{1}(t+\varepsilon)$

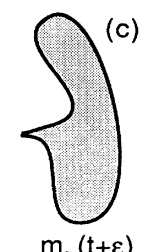

(c)

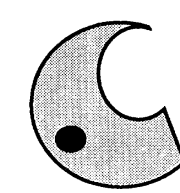

$m_{1}(t+\varepsilon) \quad m_{2}(t+\varepsilon)$

(d)

(e)

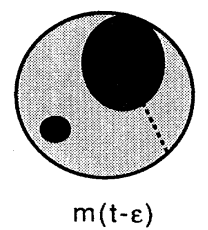

(f)

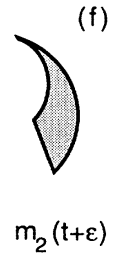

FIG. 1. Two-dimensional schematic representation of fragmentation with continuous mass loss (a)-(c) and with continuous and discrete mass loss (d)-(f). A porous homogeneous particle shown at (a) time $t=0$ (b) time $t-\epsilon$ just before fragmentation, and (c) time $t+\epsilon$ just after fragmentation as the particle falls apart, where $\epsilon$ is an infinitesimal time. A two-phase heterogeneous particle with inclusions of an explosive (heavily shaded) phase (d) initially, (e) just before fragmentation, and (f) just after fragmentation illustrates how continuous mass loss exposes and ignites an explosive inclusion at time $t$, causing fragmentation due to the violence of the explosion. For both cases, the continuous-mass-loss fraction $\lambda_{c}=[m(0)-m(t-\epsilon)] / m(0)$ measures the fractional loss in the (parent) particle mass that occurs before fragmentation and the discrete mass loss fraction $\lambda=\left[m(t-\epsilon)-m_{1}(t+\epsilon)-m_{2}(t+\epsilon)\right] / m(t-\epsilon)$ measures the fractional loss in the overall mass that occurs during fragmentation, where $m_{1}(t+\epsilon)$ and $m_{2}(t+\epsilon)$ are the masses of the daughter particles produced by the fragmentation event. For case (d)-(f), the loss of the mass of the large inclusion during fragmentation renders the fragmentation event non-massconserving so that $\lambda \neq 0$.

tion of particles in the same range, the increase in the number of particles in the range due to fragmentation of larger particles, and the change in the number of particles in the range due to continuous mass loss. To allow for discrete mass loss, we normalize $\bar{b}(x \mid y)$ according to

$$
\int_{0}^{y} x \bar{b}(x \mid y) d x=[1-\bar{\lambda}(y)] y,
$$

where the left side gives the total mass of fragments spawned by an average fragmentation event, and the right side gives the parent particle mass $y$ just before fragmentation reduced by the average mass $\bar{\lambda}(y) y$ lost at the instant of fragmentation, where $0 \leq \bar{\lambda}(y) \leq 1$. The average is over many fragmentation events of the type shown in

Fig. 1(d)-1(f). Fragmentation regimes associated with the continuous-loss fraction $\lambda_{c}$ defined as the fraction of a particle's mass lost continuously during its lifetime (see Fig. 1 caption) are discussed in Ref. 6.

The average number of fragments resulting from the fragmentation of a particle of mass $y$ is

$$
\int_{0}^{y} \bar{b}(x \mid y) d x=\overline{\mathcal{N}}(y) \text {. }
$$

Binary fragmentation $[\overline{\mathcal{N}}(y)=2]$ requires the symmetry condition $\bar{b}(x \mid y)=\bar{b}(y-x \mid y)$. The linearity of Eq. (1) reflects the regularity of the continuous and discrete loss processes; other fragmentation processes caused by repeated collisions between particles require a nonlinear rate equation. ${ }^{2}$ The mass-loss term [last term in Eq. (1)] has been used previously to describe evaporation in the context of aerosol coagulation. ${ }^{8}$

To justify Eq. (1) in the absence of fragmentation and to identify the particle current, it is useful to follow a group of particles subject to continuous mass loss. By definition, the number of particles in the mass range $[x: x+d x]$ at time $t$ is $n(x, t) d x$. After a time $d t$, the mass range of this same group of particles is

$$
[x-c(x) d t: x+d x-c(x+d x) d t]
$$

$$
=\left[x-c(x) d t: x-c(x) d t+d x\left[1-c^{\prime}(x) d t\right]\right],
$$

where the equality is valid to first order in $d x$. Neglecting fragmentation, the number of particles in the group cannot change, so that

$n(x, t) d x=n(x-c(x) d t, t+d t)\left[1-c^{\prime}(x) d t\right] d x$.

Since, to first order in $d t$,

$n(x-c(x) d t, t+d t)$

$$
=n(x, t)-c(x) \frac{\partial n(x, t)}{\partial x} d t+\frac{\partial n(x, t)}{\partial t} d t
$$

we can rewrite Eq. (4) as

$$
\frac{\partial n(x, t)}{\partial t}+\frac{\partial}{\partial x}[-c(x) n(x, t)]=0 .
$$

This equation verifies Eq. (1) in the absence of fragmentation and expresses conservation of particles, with a particle "current" $-c(x) n(x, t)$ giving the overall fractional rate of change of mass for particles of mass $x$. A derivation of Eq. (1) including fragmentation is given in Ref. 6.

The familiar equation for mass-conserving fragmentation, ${ }^{1-4}$

$$
\frac{\partial n(x, t)}{\partial t}=-a(x) n(x, t)+\int_{x}^{\infty} a(y) \bar{b}(x \mid y) n(y, t) d y,
$$

and the corresponding normalization

$$
\int_{0}^{y} x \bar{b}(x \mid y) d x=y
$$

follow from Eqs. (1) and (2) by setting $c(x)=0$ and $\bar{\lambda}(y)=0$. For binary fragmentation, this equation is equivalent to the equation used to study polymer breakup, ${ }^{1}$ 


$$
\begin{aligned}
\frac{\partial n(x, t)}{\partial t}= & -n(x, t) \int_{0}^{x} F(y, x-y) d y \\
& +2 \int_{x}^{\infty} n(y, t) F(x, y-x) d y,
\end{aligned}
$$

where $F(x, y-x)=a(y) b(x \mid y) / 2$ is the rate at which particles of mass $y$ break into two particles of mass $x$ and $y-x$, and $\int_{0}^{y} b(x \mid y) d x=2$.

\section{DISCRETE-SPACE SOLUTIONS IN ONE DIMENSION}

It is instructive to apply discrete-space continuoustime versions of Eqs. (1), (2), and (3),

$$
\begin{aligned}
& \frac{d n_{x}(t)}{d t}=-a_{x} n_{x}(t)+\sum_{y=y_{l}}^{\infty} a_{y} \bar{b}_{x \mid y} n_{y}(t) \\
&+c_{x+1} n_{x+1}(t)-c_{x} n_{x}(t), \\
& \sum_{x=x_{l}}^{x_{h}} x \bar{b}_{x \mid y}=\left(1-\bar{\lambda}_{y}\right) y, \\
& \sum_{x=x_{l}}^{x_{h}} \bar{b}_{x \mid y}=\overline{\mathcal{N}}_{y},
\end{aligned}
$$

to simple one-dimensional fragmentation processes. Here, $n_{x}(t)$ denotes the number of particles of length (mass) $x$ at time $t$, with $x$ restricted to positive integer values. Values of $y_{l}, x_{l}$, and $x_{h}$ are chosen below to correspond to various discrete binary processes with $\overline{\mathcal{N}}_{y}=2$.

For "random scission" in one dimension, an initially infinite chain is subject to mass-conserving random cutting events only at integer positions along the chain, called "junctions." Correspondingly, the length of a daughter chain resulting from the fragmentation of a parent chain of length $y$ must satisfy $1 \leq x \leq y-1$, so that $x_{l}=1, x_{h}=y-1$, and $y_{l}=x+1$. Since the scission is random, all unbroken junctions are equally likely to break, so the fragmentation rate for a parent chain of length $y$ is the number of unbroken junctions in the chain, $a_{y}=y-1$, and $\vec{b}_{x \mid y}$ is independent of $x$ over the relevant range of $x$. With $\bar{b}_{x \mid y}=2 /(y-1)$ following from Eq. (9) and with no mass loss $\left(c_{x}=0\right)$, Eq. (7) takes the familiar form ${ }^{1}$

$$
\frac{d n_{x}}{d t}=-(x-1) n_{x}+2 \sum_{y=x+1}^{\infty} n_{y} .
$$

As expected, Eq. (8) implies that mass is conserved; $\bar{\lambda}_{y}=0$.

An important one-dimensional discrete process, similar to random scission, but which does not conserve mass, is "random bond annihilation". A "bond" is a unit line segment joining two adjacent integer values of $x$, so that annihilation (removal) of a bond implies a unit reduction in the overall mass. Here, bonds can be randomly annihilated at the chain surface (at one of the two end bonds of a continuous chain) or anywhere within the chain interior with equal probability. The surface recession terms [the last two terms in Eq. (7)] are unnecessary if annihilation of any bond, including a surface bond, is deemed a fragmentation event. In this case, the fragmentation rate is the length of the chain, $a_{y}=y$, and the possible daughter lengths satisfy $0 \leq x \leq y-1$. Correspondingly, we obtain $x_{l}=0, x_{h}=y-1, y_{l}=x+1, \vec{b}_{x \mid y}=2 / y$, and $c_{x}=0$, leaving

$$
\frac{d n_{x}}{d t}=-x n_{x}+2 \sum_{y=x+1}^{\infty} n_{y},
$$

which differs from Eq. (10) by a single term. Equation (8) supplies the expected discrete loss fraction, $\bar{\lambda}_{y}=1 / y$.

For a more natural approach to random annihilation in one dimension, the surface recession terms can describe annihilation of surface bonds while the fragmentation terms describe only the annihilation of interior bonds. The continuous-mass-loss rate $c_{y}=2$ accounts for the annihilation of (two) end bonds on a parent chain of length $y$, while the fragmentation rate $a_{y}=y-2$ accounts for the annihilation of interior bonds, with possible daughter chain lengths satisfying $1 \leq x \leq y-2$. According, $x_{l}=1$, $x_{h}=y-2$, and $y_{l}=x+2$. Since random interior-bond annihilation always leads to fragmentation into two smaller chains $\left(\overline{\mathcal{N}}_{y}=2\right)$, Eq. (9) implies the $x$-independent distribution $\bar{b}_{x \mid y}=2 /(y-2)$, Eq. (8) supplies the expected value $\bar{\lambda}_{y}=1 / y$, and Eq. (7) yields Eq. (11). Thus, this approach constitutes a specific confirmation of Eq. (1) involving both continuous and discrete mass loss. The solution $n_{x}(t)=\left(1-e^{-t}\right)^{2} e^{-x t}$ of Eq. (11) and correspondences with one-dimensional bond percolation are discussed in Ref. 6.

The two approaches to random annihilation illustrate the mutual exclusiveness of the fragmentation and surface-recession terms in Eq. (1). In each approach, annihilation events are described by either the fragmentation or the surface-recession term, but not both. Note that the surface-recession term cannot describe interior bond annihilation, since the annihilation of an interior bond in a one-dimensional chain always leads to fragmentation.

\section{EQUAL-FRAGMENT RECESSION}

A spatially continuous deterministic “equal-fragment recession" process with discrete mass loss yields exact solutions. At time $T$, a particle of initial mass $m_{0}$ breaks into a fixed number $N_{1}=\mathcal{N}$ of fragments each of equal mass $m_{1}=(1-\lambda) m_{0} / \mathcal{N}$, where $\lambda$ is the fixed discrete loss fraction. At time $2 T$, each of these fragments breaks into $\mathcal{N}$ subfragments each of mass $m_{2}=(1-\lambda) m_{1} / \mathcal{N}$, for a total of $N_{2}=\mathcal{N}^{2}$ subfragments. Continuing similarly, the recurrence relations $m_{i+1}=v m_{i}$ [with $\nu=(1-\lambda) / \mathcal{N}$ ] and $N_{i+1}=\mathcal{N} N_{i}$ yield the useful relations $m_{i}=v^{i} m_{0}$ and $N_{i}=\mathcal{N}^{i}$.

Since there is no continuous mass loss between fragmentation events for equal-fragment recession, the number and the individual masses of particles present at time $t$ are piecewise uniform and can be written as $N(t)=\sum_{i=0}^{\infty} \eta_{i}(t) N_{i}$ and $m(t)=\sum_{i=0}^{\infty} \eta_{i}(t) m_{i}$, where $\eta_{i}(t)=1$ for $i T<t<(i+1) T$, and $\eta_{i}(t)=0$ otherwise. The function $\eta_{i}(t)$ can be described by the step function 
$\Theta(t)$ as $\eta_{i}(t)=\Theta(t-i T)-\Theta(t-(i+1) T)$. The recurrence relations require each daughter spawned by a parent particle of mass $y$ to have mass $x=v y$. The corresponding $\bar{b}(x \mid y)=\mathcal{N} \delta(x-v y)$ satisfies Eqs. (2) and (3) with $\bar{\lambda}(y)=\lambda$ and $\overline{\mathcal{N}}(y)=\mathcal{N}$. The quantity $a(x) d t$ is defined as the fraction of particles of mass $x$ that undergo fragmentation in time $d t$. Consequently, since all parti- cles of a particular size undergo fragmentation at the same time for this deterministic process (rather than at different times for randomized processes involving ensembles of particles, Sec. V), $a(x) d t=1$ if $d t$ encloses the time of a fragmentation event and $a(x) d t=0$ otherwise. The proposed solution $n(x, t)=N(t) \delta(x-m(t))$ is verified by substitution into Eq. (1) [with $c(x)=0$ ];

$$
\begin{aligned}
d n(x, t) & =-[a(x) d t] n(x, t)+\int_{x}^{\infty}[a(y) d t][\mathcal{N} \delta(x-v y)] n(y, t) d y \\
& =-[a(x) d t] n(x, t)+(\mathcal{N} / v)[a(x / v) d t] n(x / v, t)
\end{aligned}
$$

If $d t$ does not enclose a fragmentation event, the left side of Eq. (12) vanishes due to the constancy of $N(t)$ and $m(t)$, and the right side vanishes because $a(x) d t=0$. Between $t=i T-d t / 2$ and $t=i T+d t / 2$, the time interval $d t$ encloses a fragmentation event and Eq. (12) becomes

$$
n(x, i T+d t / 2)-n(x, i T-d t / 2)=-n(x, i T-d t / 2)+(\mathcal{N} / v) n(x / v, i T-d t / 2) .
$$

The recurrence relations imply that

$$
\begin{aligned}
n(x / v, i T-d t / 2) & =N(i T-d t / 2) \delta(x / v-m(i T-d t / 2)) \\
& =\mathcal{N}^{-1} N_{i} \delta\left(x / v-m_{i} / v\right) \\
& =(v / \mathcal{N}) n(x, i T+d t / 2),
\end{aligned}
$$

hence $n(x, t)=N(t) \delta(x-m(t))$ is an exact solution.

Equal-fragment recession can also be solved exactly for mass-conserving fragmentation events. In this case, the loss fraction $\lambda$ is removed continuously between fragmentation events, and the particle mass $m(t)$ $=\sum_{i=0}^{\infty} \eta_{i}(t) m_{i}[1-\lambda(t-i T) / T]$ is piecewise linear. In this way, $\lambda$ is a "continuous-loss fraction," the fraction of the initial mass of a particle lost continuously during its lifetime. Clearly, $m(t)$ agrees with the previous form just after fragmentation events, and $N(t)=\sum_{i=0}^{\infty} \eta_{i}(t) N_{i}$ is the same as before. The form of $\bar{b}(x \mid y)=\mathcal{N} \delta(x-y / \mathcal{N})$ [consistent with Eqs. (2) and (3)] reflects the conservation of mass at fragmentation events. Although $a(x)$ is the same as before, $c(x)=c(m(t))$ now gives the continuousmass-loss rate between fragmentation events of a particle of mass $m(t)$, so that $c(m(t))=-[d m(t) / d t]_{t \neq i T}$ $=(\lambda / T) \sum_{i=0}^{\infty} \eta_{i}(t) m_{i}$. Evidently, $c(x)$ is independent of $x$ between fragmentation events (for $t \neq i T$ ) reflecting the determinisitic process under consideration. The proposed solution $n(x, t)=N(t) \delta(x-m(t))$ differs from before only in the form of $m(t)$. Between fragmentation events, the fragmentation terms play no role as before, $d N / d t=0$, and Eq. (1) becomes

$N(t) \frac{\partial}{\partial t} \delta(x-m(t))=-\frac{d m(t)}{d t} N(t) \frac{\partial}{\partial x} \delta(x-m(t))$,

thus verifying the solution between fragmentation events. At fragmentation events, noting that $m(i T-d t / 2)$ $=\mathcal{N} m(i T+d t / 2)$ and that $c(m(i T))=0$, the analysis proceeds much as before and verifies the solution.

Thus, our exact forms for $n(x, t)$ for equal-fragment recession with discrete and continuous mass loss each satisfy the rate equation and agree with each other just after fragmentation events. Accordingly, we observe that problems with continuous mass loss and mass-conserving fragmentation events can be well approximated by ascribing all the mass loss to discrete fragmentation events as long as the relevant loss fractions are small.

A deterministic "unequal-fragment" binary recession process leads to a distribution of particle masses. The unequal-fragment process requires, during each time interval $T$, that a particle of initial mass $m$ lose a mass $\lambda m$ to surface recession. After an elapsed time $T$, the particle breaks into two particles of masses $\xi(1-\lambda) m / 2$ and $(1-\xi)(1-\lambda) m / 2$, with $0<\xi<1$. Although binomial coefficients help to provide exact solutions to this process, they fail to further illuminate the physics of fragmentation with mass loss.

\section{SCALING}

It is instructive to study scaling for the general power law forms $a(x)=\widehat{a} x^{\alpha}$ and $c(x)=\widehat{c} x^{\gamma}$. For the study, it is convenient to use dimensionless variables. By choosing an arbitrary mass scale $\hat{x}$, we define dimensionless (primed) variables by the relations $x=\hat{x} x^{\prime}$, $t=\left(\widehat{a} \hat{x}^{\alpha}\right)^{-1} t^{\prime} \equiv \hat{t} t^{\prime}, \quad a(x)=\hat{t}^{-1} a^{\prime}\left(x^{\prime}\right), \quad \bar{b}(x \mid y)$ $=\hat{x}^{-1} \bar{b}^{\prime}\left(x^{\prime} \mid y^{\prime}\right), \quad c(x)=\hat{x} \hat{t}{ }^{-1} c^{\prime}\left(x^{\prime}\right), \quad n(x, t)=n\left(\hat{x} x^{\prime}, \hat{t} t^{\prime}\right)$ $=\hat{x}^{-1} n^{\prime}\left(x^{\prime}, t^{\prime}\right), \bar{\lambda}(y)=\bar{\lambda}^{\prime}\left(y^{\prime}\right)$, and $\overline{\mathcal{N}}(y)=\overline{\mathcal{N}}^{\prime}\left(y^{\prime}\right)$. Substituting these relations leaves Eqs. (1)-(3) unchanged except that all variables are primed. By henceforth dropping the primes and treating all quantities as dimensionless, we can use Eqs. (1)-(3) with $a(x)=x^{\alpha}$ and $c(x)=\epsilon x^{\gamma}$, where the dimensionless ratio $\epsilon=(\widehat{c} / \widehat{a}) \hat{x}^{\gamma-\alpha-1}$ governs the importance of continuous mass loss relative to fragmentation.

The mass-loss exponent $\gamma$ governs the lifetime $T_{s}$ of a 
particle subject to surface recession in the absence of fragmentation. The equation $c(m(t))=\epsilon m^{\gamma}(t)$ $=-d m(t) / d t$ yields the time-dependent mass of a particle of initial mass $m_{0}$,

$$
m(t)=m_{0}\left[1+\epsilon(\gamma-1) m_{0}^{-1} t\right]^{-1 /(\gamma-1)} .
$$

Thus, $\gamma<1$ requires that the mass vanish in a finite particle lifetime $T_{s}=\epsilon^{-1}(1-\gamma)^{-1} m_{0}^{1-\gamma}, \gamma=1$ gives an exponential decay $m(t)=m_{0} e^{-\epsilon t}$, and $\gamma>1$ gives an $m_{0^{-}}$ independent power-law decay at long times $m(t)=[\epsilon(\gamma-1) t]^{-1 /(\gamma-1)}$. Infinite particle lifetimes for $\gamma>1$ reflect the small mass-loss rates $c(x)=\epsilon x^{\gamma}$ for small particle masses $x$.

The relation $\gamma=\alpha+1$ and the restricted form $\bar{b}(x \mid y)=h(x / y) / y$ yield a scale-invariant form ${ }^{6}$ of Eq. (1),

$$
\begin{aligned}
\frac{\partial n(x, t)}{\partial t}= & -x^{\alpha} n(x, t)+\int_{x}^{\infty} y^{\alpha-1} h(x / y) n(y, t) d y \\
& +\epsilon \frac{\partial}{\partial x}\left[x^{\alpha+1} n(x, t)\right]
\end{aligned}
$$

with a simpler form for $\epsilon=\widehat{c} / \widehat{a}$. By definition, $\bar{b}(x \mid y) d x$ is the probability that one of the daughters from a parent of mass $y$ has mass between $x$ and $x+d x$. Defining the daughter fraction as $r=x / y$, this probability reduces to $\bar{b}(x \mid y) d x=h(x / y) d x / y=h(r) d r$, the probability of finding a daughter fraction between $r$ and $r+d r$. Thus, $h(r)$ is the distribution of daughter fractions. That this distribution is independent of the parent mass reflects the essence of scaling. The normalization condition [Eq. (2)] and Eq. (3) now take the simpler forms

$$
\int_{0}^{1} r h(r) d r=1-\bar{\lambda}
$$

and

$$
\int_{0}^{1} h(r) d r=\overline{\mathcal{N}}
$$

with a $y$-independent average discrete loss fraction $\bar{\lambda}$ and average number of fragments $\overline{\mathcal{N}}$. Thus, the scaling regime is a set of conditions under which all physical properties are scale invariant: the particle mass distribution, apart from trivial spatial rescaling, looks the same at all times.

To determine the conditions necessary for runaway fragmentation, or "shattering," we define the moments ${ }^{2}$ $M_{\mu}(t)=\int_{0}^{\infty} x^{\mu} n(x, t) d x$ and $L_{\mu}=\int_{0}^{1} r^{\mu} h(r) d r$. These allow us to write the $\mu$ th moment of Eq. (14) as

$$
\dot{M}_{\mu}=-\left(1-L_{\mu}+\mu \epsilon\right) M_{\mu+\alpha},
$$

where the overdot signifies a time derivative and $\left.\epsilon x^{\mu+\alpha+1} n(x, t)\right|_{0} ^{\infty}=0$. The rate of change of the total mass of the system is given by Eq. (17) with $\mu=1$. Setting $\mu=1+\Delta$ with positive $\Delta \rightarrow 0$, using the first-order expansion $L_{1+\Delta}=L_{1}-\Delta K$ with $K=-\left(d L_{\mu} / d \mu\right)_{\mu=1}>0$, and using Eq. (15), we obtain

$\dot{M}_{1+\Delta}=-(\bar{\lambda}+\epsilon) M_{1+\Delta+\alpha}-\Delta(K+\epsilon) M_{1+\Delta+\alpha}$.

The first term on the right side gives the expected discrete and continuous loss rates. The second term gives shattering mass loss if $M_{1+\Delta+\alpha}$ diverges as $1 / \Delta$ or faster. Setting $\mu=1-\alpha$ in Eq. (17) yields a second useful equation,

$$
\dot{M}_{1-\alpha}=-\left[1-L_{1-\alpha}+(1-\alpha) \epsilon\right] M_{1} .
$$

For $\bar{\lambda}=\epsilon=0$, assuming no shattering mass loss in Eq. (18) implies that $M_{1}=$ const. Since $L_{\mu}<1-\bar{\lambda}$ for $\mu>1$ [by definition and by Eq. (15)], Eq. (19) implies that $M_{1-\alpha}$ vanishes at a finite time only for $\alpha<0$. That $n(x, t)$ consequently vanishes at a finite time contradicts the assumption that shattering mass loss is absent. Thus, $\alpha<0$ implies shattering ${ }^{2}$ for fragmentation presumably absent of mass loss, with a time-dependent total mass obeying $\dot{M}_{1+\Delta}=-\Delta K M_{1+\Delta+\alpha}$ as $\Delta \rightarrow 0$. The shattering mass loss is due to runaway fragmentation, a cascade of fragmentation events which reduces finite-mass particles to infinite numbers of zero-mass particles in a finite time. ${ }^{3}$

For fragmentation with mass loss (with $\bar{\lambda} \neq 0$ and/or $\epsilon \neq 0$ ), we have found no contradiction of the assumption that shattering mass loss is absent. By Eq. (18), any shattering mass loss would be negligible compared to discrete and/or continuous mass loss since the second term vanishes as $\Delta$ or faster relative to the first, so that

$$
\dot{M}_{1}=-(\bar{\lambda}+\epsilon) M_{1+\alpha} \text {. }
$$

Thus, even though runaway fragmentation occurs for $\sigma \geq 0$ and $\alpha<0$ (Ref. 6), we expect that discrete and continuous mass loss account for all mass loss and precludes the mass loss normally associated with shattering.

To study scaling for $\alpha>0$, it is helpful to substitute the scale-invariant "scaling" form"

$$
n(x, t)=t^{\delta} f\left(x t^{z}\right)
$$

with $z=1 / \alpha$ into Eq. (14),

$$
\begin{aligned}
\delta f(\xi)+\alpha^{-1} \xi f^{\prime}(\xi)= & -\xi^{\alpha} f(\xi) \\
& +\int_{\xi}^{\infty} \eta^{\alpha-1} h(\xi / \eta) f(\eta) d \eta \\
& +\epsilon \frac{d}{d \xi}\left[\xi^{\alpha+1} f(\xi)\right],
\end{aligned}
$$

where $\xi=x t^{z}$ is the scaling variable. Although Eq. (14) is scale invariant, solutions to Eq. (14) are not necessarily scale invariant, in contrast with Eq. (22). Defining the $\mu$ th moment of the scaling function as $\rho_{\mu}=\int_{0}^{\infty} \xi^{\mu} f(\xi) d \xi$, Eq. (20) reduces to

$$
\dot{M}_{1}=-(\bar{\lambda}+\epsilon) t^{\delta-2 z-1} \rho_{1+\alpha} .
$$

For $h(r)=2$, Eq. (22) yields the asymptotic form $f(\xi)=\xi^{-\zeta}$ for large $\xi$, with $\xi$ in the range $1<\xi-\alpha<2$ for $\infty>\epsilon>0$. Since $h(r)=2 \mu^{-1}(\mu+2)\left(1-r^{\mu}\right)$ with $\mu>0$ behaves similarly, we infer that finite $\epsilon$ violates scaling generally at large $\xi$. For $\epsilon \rightarrow \infty$, separation of variables in Eq. (14) yields a general solution for continuous mass loss in the absence of fragmentation,

$$
n(x, t)=x^{-\alpha-1} \int_{-\infty}^{\infty} A(\omega) \exp \left(\omega \epsilon^{-1} \alpha^{-1} x^{-\alpha}\right) e^{-\omega t} d \omega,
$$

which violates scaling entirely. Setting $\epsilon=0$ in Eq. (22) gives an equation for fragmentation with discrete mass loss only, which yields explicit scaling solutions and gen- 
eral asymptotic scaling forms (below). In conclusion, we generally expect scaling for $\alpha>0$ and $\xi<\xi_{c}(\epsilon)$, where $\xi_{c} \rightarrow \infty$ as $\epsilon \rightarrow 0$ and $\xi_{c} \rightarrow 0$ as $\epsilon \rightarrow \infty$.

We now derive universal asymptotic forms of the scaling function for $\alpha>0$ by generalizing methods ${ }^{2}$ developed for mass-conserving fragmentation. We can use the definition of $\rho_{\mu}$ to write $M_{\mu}=\int_{0}^{\infty} x n(x, t) d x$ $=t^{\delta-(\mu+1) z} \rho_{\mu}$. Likewise, rewriting Eq. (17) yields

$$
\rho_{\mu+\alpha}=\frac{\delta-(\mu+1) / \alpha}{L_{\mu}-\epsilon \mu-1} \rho_{\mu} .
$$

For the general power-law decay $h(r) \sim r^{\nu}$ for small $r, L_{\mu}$ diverges for $\mu<\mu_{c}=-1-v$. Consequently, for $\mu$ approaching $\mu_{c}$ from above, Eq. (24) yields, to lending order,

$$
\rho_{\mu}=L_{\mu} \frac{\rho_{\mu_{c}+\alpha}}{\delta-\left(\mu_{c}+1\right) / \alpha} \sim \int_{0}^{1} r^{\mu} h(r) d r
$$

for finite $\epsilon$. Since only small arguments are relevant to this integral, $f(\xi)$ and $h(r)$ share the same behavior (see definition of $\rho_{\mu}$ ), so the resulting small- $\xi$ asymptotic form

$$
f(\xi) \sim \xi^{v}
$$

is independent of $\epsilon$. Although forms of $h(r)$ that vanish below some small finite cutoff $r_{0}$ could also be considered, the applications of interest to us require the power-law decay.

To identify a large- $\xi$ universal scaling form, we substitute $\mu=j \alpha$ into Eq. (24) to find

$$
\rho_{i \alpha}=\prod_{j=1}^{i-1} \frac{j+z-\delta}{1-L_{j \alpha}+\epsilon j \alpha} \rho_{\alpha} .
$$

With the general form $h(r)=h(1)+O\left(\left(1-r^{\beta}\right)\right)$ near $r=1$, we obtain $L_{j \alpha}=h(1) /(j \alpha+1)$ for large $j$. With $\epsilon=0$, the $i$ dependence of $\rho_{i \alpha}$ follows as

$$
\rho_{i \alpha} \sim \Gamma(i+z-\delta) \Gamma(i+z) / \Gamma(i+z-z h(1))
$$

and Sterling's formula yields

$$
\rho_{\mu} \sim(\mu z)^{z[\mu+h(1)+1]-\delta-1 / 2} e^{-\mu z} .
$$

For $h(r)=2$ and $\epsilon=0$, the known exact solution ${ }^{2}$ $f^{\text {exact }}(\xi)=\exp \left(-\xi^{\alpha}\right)$ yields

$$
\rho_{\mu}^{\mathrm{exact}}=z \Gamma(\mu z+z) \sim(\mu z)^{\mu z+z-1 / 2} e^{-\mu z} .
$$

Consequently,

$$
\rho_{\mu} \sim \rho_{\mu+h(1)-\delta \alpha}^{\mathrm{exact}}
$$

implies the large- $\xi<\xi_{c}$ asymptotic form

$$
f(\xi) \sim \xi^{h(1)-\delta \alpha} \exp \left(-\xi^{\alpha}\right)
$$

for $\epsilon=0$. Since the derivation requires small $\epsilon j \alpha$ compared to $1-L_{j \alpha}$, the moments with $j \rightarrow \infty$ which dominate $f(\xi)$ as $\xi \rightarrow \infty$ violate Eq. (26) for finite $\epsilon$, reflecting the violation of scaling ${ }^{6}$ at large $\xi$. However, for small $\epsilon$, moments with large $j<j_{c}=(\epsilon \alpha)^{-1}$ satisfy $\epsilon j \alpha<1-L_{j \alpha}$. Equation (26) is therefore expected to apply for small $\epsilon$ and large $\xi<\xi_{c}$, with $\xi_{c} \rightarrow \infty$ as $\epsilon \rightarrow 0$.
Explicit solutions follow by generalizing results for mass-conserving fragmentation. ${ }^{1,3}$ For an intrinsically discrete loss process $(\epsilon=0)$ with a uniform distribution $h(r)=2(1-\bar{\lambda})$ satisfying Eq. (15), Eq. (22) yields the exact scaling solution $\delta=2(1-\bar{\lambda}) / \alpha$ and $f(\xi)=\exp \left(-\xi^{\alpha}\right)$ for all $\xi$, so that

$$
n(x, t)=t^{2(1-\bar{\lambda}) / \alpha} \exp \left(-x^{\alpha} t\right) .
$$

This solution agrees with the asymptotic scaling forms, Eqs. (25) and (26), with $v=0$. It also gives the explicit value $\delta \alpha=2(1-\bar{\lambda})<2$ relevant to mass loss.

The general solution of Eq. (14) can also be found for the problem just discussed;

$$
\begin{gathered}
n(x, t)=\exp \left(-x^{\alpha} t\right) \int_{0}^{\infty} A(s)(1+t / s)^{2(1-\bar{\lambda}) / \alpha} \\
\times \exp \left(-s x^{\alpha}\right) d s
\end{gathered}
$$

where $A(s)$ reflects arbitrary initial conditions. This solution approaches the scaling solution [Eq. (27)] for large $t$ (apart from an overall constant factor), as expected.

Solutions for the power-law distribution $h(r)=(v+2)(1-\bar{\lambda}) r^{v}$ with $v>-2$ and $\epsilon=0$ follow from the transformation $x=\widetilde{x}^{2 /(v+2)}$ and $n(x, t)=x^{v} \widetilde{n}(\widetilde{x}, t)$. Substituting these relations into Eq. (14) yields

$$
\begin{aligned}
\frac{\partial \widetilde{n}(\tilde{x}, t)}{\partial t}=- & \widetilde{x}^{\tilde{\alpha}} \widetilde{n}(x, t)+2(1-\bar{\lambda}) \\
& \times \int_{\widetilde{x}}^{\infty} \widetilde{y}^{\tilde{\alpha}-1} \widetilde{n}(\widetilde{y}, t) d \widetilde{y} .
\end{aligned}
$$

With $\widetilde{\alpha}=2 \alpha /(v+2)$, this equation corresponds to the equation for uniform distribution $h(r)=2(1-\bar{\lambda})$ discussed above. Consequently, Eqs. (27) and (28) immediately yield the scaling solution

$$
n(x, t)=x^{v} t^{(v+2)(1-\bar{\lambda}) / \alpha} \exp \left(-x^{\alpha} t\right)
$$

and the general solution

$$
\begin{aligned}
& n(x, t)= x^{v} \exp \left(-x^{\alpha} t\right) \\
& \times \int_{0}^{\infty} A(s)(1+t / s)^{(v+2)(1-\bar{\lambda}) / \alpha} \\
& \quad \times \exp \left(-s x^{\alpha}\right) d s .
\end{aligned}
$$

The corresponding scaling quantities $\delta=(v+2)(1$ $-\bar{\lambda}) / \alpha-v / \alpha$ and $f(\xi)=\xi^{v} \exp \left(-\xi^{\alpha}\right)$ agree with the large- and small- $\xi$ scaling forms, Eqs. (25) and (26).

Alternate scaling forms of $n(x, t)$ are sometimes useful. The form

$$
n(x, t)=x^{-\delta \alpha} F\left(x t^{z}\right)
$$

follows from the scaling relation $\alpha=1 / z$, Eq. (21), and the definition $F(\xi)=\xi^{\delta \alpha} f(\xi)$. This form is analogous to the scaling hypothesis for percolation. ${ }^{9}$ When $h(r) \sim r^{v}$ for small $r$, we can write

$$
n(x, t)=t^{w} x^{\tau} g\left(x t^{z}\right),
$$

where $\omega=\delta+v z, \tau=v$, and $g(\xi) \sim \xi^{-v} f(\xi) \rightarrow$ const as $\xi \rightarrow 0$ [see Eq. (25)]. 


\section{CONCLUSIONS}

We have expanded the theory of linear fragmentation with mass loss by finding solutions for random, deterministic, and spatially discrete systems, and by deriving asymptotic scaling forms. We are in the process of obtaining numerical solutions to determine the behavior of the system in regimes which are intractible analytically. It would also be useful to compare the predictions of the rate equation with experiments. For this purpose, it may be useful to study more complicated forms of $h(r)$.

\section{ACKNOWLEDGMENTS}

We gratefully acknowledge helpful discussions with Alan Kerstein and Richard Treat. This work was supported by the West Virginia University Energy and Water Research Center, the Pittsburgh Supercomputing Center, and by U.S. Department of Energy Grant No. DE-FG22-89PC89791.
${ }^{1}$ T. A. Bak and K. Bak, Acta Chem. Scand. 13, 1997 (1959); M. Aizenman and T. A. Bak, Commun. Math. Phys. 65, 203 (1979); R. M. Ziff and E. D. McGrady, Macromolecules 19, 2513 (1986)

${ }^{2} Z$. Cheng and S. Redner, Phys. Rev. Lett. 60, 2450 (1988).

${ }^{3}$ E. D. McGrady and R. M. Ziff, Phys. Rev. Lett. 58, 892 (1987); R. M. Ziff and E. D. McGrady, J. Phys. A 18, 3027 (1985).

${ }^{4}$ A. R. Kerstein (private communication).

${ }^{5}$ D. Dunn-Rankin and A. R. Kerstein, Combust. Flame 69, 193 (1987), and references therein; C. A. Sundback, J. M. Beér, and A. F. Sarofim, Proceedings of the 20th International Sym- posium on Combustion (The Combustion Institute, Pittsburgh, 1985), p. 1495.

${ }^{6}$ B. F. Edwards, M. Cai, and H. Han, Phys. Rev. A 41, 5755 (1990).

${ }^{7}$ M. Sahimi and T. T. Tsotsis, Phys. Rev. Lett. 59, 888 (1987).

${ }^{8}$ T. W. Peterson, F. Gelbard, and J. M. Seinfeld, J. Colloid Interface Sci. 63, 426 (1978).

${ }^{9}$ D. Stauffer, Introduction to Percolation Theory (Taylor and Francis, Philadelphia, 1985), Chap. 2; H. Nakanishi and H. E. Stanley, Phys. Rev. B 22, 2466 (1980). 